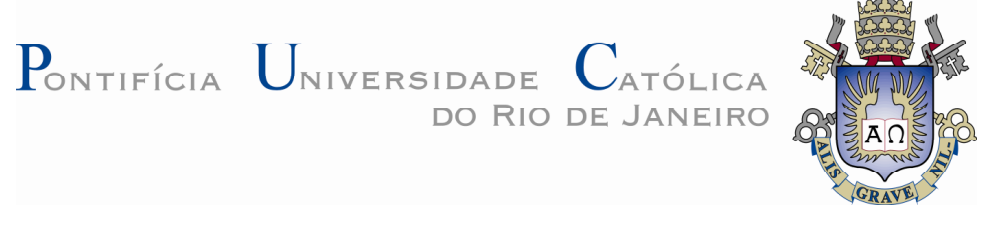

Alexandre Ormiga Galvão Barbosa

\title{
CONTROLE DE UM MANIPULADOR ROBÓTICO ATRAVÉS DE UMA INTERFACE CÉREBRO MÁQUINA NÃO-INVASIVA COM APRENDIZAGEM MÚTUA
}

\begin{abstract}
Dissertação de Mestrado
Dissertação apresentada ao Programa de PósGraduação em Engenharia Mecânica da PUC-Rio como requisito parcial para obtenção do título de Mestre em Engenharia Mecânica.
\end{abstract}

Orientador: Prof. Marco Antonio Meggiolaro

Rio de Janeiro

Agosto de 2010 


\title{
CONTROLE DE UM MANIPULADOR ROBÓTICO ATRAVÉS \\ DE UMA INTERFACE CÉREBRO MÁQUINA NÃO-INVASIVA COM APRENDIZAGEM MÚTUA
}

\begin{abstract}
Dissertação apresentada como requisito parcial para obtenção do grau de Mestre pelo Programa de PósGraduação em Engenharia Mecânica do Centro Técnico Científico da PUC-Rio. Aprovada pela Comissão Examinadora abaixo assinada.
\end{abstract}

Prof. Marco Antonio Meggiolaro Orientador Departamento de Engenharia Mecânica - PUC-Rio

Profa. Marley Maria Bernardes Rebuzzi Vellasco Departamento de Engenharia Elétrica - PUC-Rio

Prof. Luciano Luporini Menegaldo Departamento de Engenharia Mecânica e de Materiais - IME

Prof. José Eugenio Leal Coordenador Setorial do Centro Técnico Científico - PUC-Rio 
Todos os direitos reservados. É proibida a reprodução total ou parcial do trabalho sem autorização da universidade, do autor e do orientador.

\section{Alexandre Ormiga Galvão Barbosa}

Graduou-se em Engenharia Elétrica com ênfase em Sistemas Eletrônicos (Universidade Estadual do Rio de Janeiro) em 2008. No mesmo ano iniciou seu mestrado na área de Mecânica Aplicada na Pós-Graduação da PUC-Rio. Participa desde 2007 de projetos de robótica no Laboratório de Engenharia de Controle e Automação (LECA). Suas áreas de interesse abrangem robótica, bioengenharia e inteligência artificial.

Ficha Catalográfica

Barbosa, Alexandre Ormiga Galvão

Controle de um manipulador robótico através de uma interface cérebro máquina não-invasiva com aprendizagem mútua / Alexandre Ormiga Galvão Barbosa ; orientador: Marco Antonio Meggiolaro. 2010.

124 f. : il. (color.) ; $30 \mathrm{~cm}$

Dissertação (mestrado)-Pontifícia Universidade Católica do Rio de Janeiro, Departamento de Engenharia Mecânica, 2010.

Inclui bibliografia

1. Engenharia mecânica - Teses. 2. Interface cérebro máquina. 3. Redes neurais. 4. Eletroencefalograma. 5. Manipulador robótico. 6. Condicionamento operante. 7. Transformada Wavelet. 8. Filtros digitais. 9. Reabilitação. I. Meggiolaro, Marco Antonio. II. Pontifícia Universidade Católica do Rio de Janeiro. Departamento de Engenharia Mecânica. III. Título. 
Aos meus pais, Conrado e Denise. 


\section{Agradecimentos}

À FAPERJ, pelo apoio financeiro;

À PUC-Rio, pelo seu excelente corpo acadêmico que me deu as bases para este estudo;

À UERJ pela base acadêmica para ingressar num programa de pós-graduação;

Ao orientador Marco Antonio Meggiolaro, pela oportunidade, incentivo, orientação e amizade, dentro e fora do centro acadêmico;

Ao professor Mauro Speranza Neto, pela oportunidade de conhecer o laboratório de engenharia de controle e automação, e pelo incentivo durante os anos de pesquisa.

Ao meu pai, que contribuiu, desde muito cedo, de forma imensurável para minha formação como engenheiro, e me ensinou a nunca desistir dos meus sonhos, não importando o quanto eles pareçam difíceis.

À minha mãe, por me ensinar a ser humano.

Às minhas irmãs, por todo apoio, incentivo e amor durante minha vida.

À minha namorada Dianna de Paula Renner, por compartilhar meus momentos de alegria e tristeza, me dando forças para nunca desanimar.

Aos alunos de graduação e pós-graduação que diariamente trabalham no laboratório e me ajudaram tanto para a conclusão desse trabalho.

Aos meus amigos, pela descontração e alegria. 


\section{Resumo}

Barbosa, Alexandre Ormiga Galvão; Meggiolaro, Marco Antonio. Controle de um manipulador robótico através de uma interface cérebro máquina não-invasiva com aprendizagem mútua. Rio de Janeiro, 2010. 124p. Dissertação de Mestrado - Departamento de Engenharia Mecânica, Pontifícia Universidade Católica do Rio de Janeiro.

Esse trabalho apresenta o desenvolvimento de uma interface cérebromáquina (Brain Machine Interface - BMI) como um meio alternativo de comunicação para uso na robótica. O trabalho engloba o projeto e construção de um eletroencefalógrafo (EEG), assim como o desenvolvimento de todos os algoritmos computacionais e demais técnicas necessárias para o reconhecimento de atividades mentais. A interface cérebro-máquina desenvolvida é utilizada para comandar os movimentos de um manipulador robótico MA2000, associando quatro atividades mentais distintas a quatro movimentos do manipulador. A interface baseia-se na análise de sinais eletroencefalográficos, extraindo desses características que podem ser classificadas como uma atividade mental específica. Primeiramente os sinais EEG são pré-processados, filtrando-se os ruídos indesejados, utilizando filtros espaciais para o aumento da resolução espacial do escalpe, e extraindo-se características relevantes à classificação das atividades mentais. Em seguida, diferentes modelos de classificadores são propostos, avaliados e comparados. Por último, duas implementações dos classificadores são propostas para aumentar o índice de comandos corretos para o manipulador. Em uma das implementações, obtiveram-se taxas de acerto de até $91 \%$ dos comandos, enquanto a taxa de comandos incorretos chegou ao mínimo de $1.25 \%$ após 400 tentativas de controle do manipulador.

\section{Palavras-chave}

Interface Cérebro Máquina; Redes Neurais; Eletro-encefalograma; Manipulador Robótico; Condicionamento operante; Transformada Wavelet; Filtros digitais; Reabilitação. 


\section{Abstract}

Barbosa, Alexandre Ormiga Galvão; Meggiolaro, Marco Antonio (Advisor). CONTROL OF A ROBOTIC ARM THROUGH A BRAIN MACHINE INTERFACE WITH MUTUAL LEARNING. Rio de Janeiro 2010. 124p. MSc Dissertation - Departamento de Engenharia Mecânica, Pontifícia Universidade Católica do Rio de Janeiro.

This work presents the development of a brain machine interface as an alternative communication channel to be used in Robotics. It encompasses the implementation of an electroencephalograph (EEG), as well as the development of all computational methods and necessary techniques to identify mental activities. The developed brain machine interface (BMI) is applied to activate the movements of a MA2000 robotic arm, associating four different mental activities to robotic arm commands. The interface is based on EEG signal analyses, which extract features that can be classified as specific mental activities. First, a signal preprocessing is performed from the EEG data, filtering noise, using a spatial filter to increase the scalp signal resolution, and extracting relevant features. Then, different classifier models are proposed, evaluated and compared. Finally, two implementations of the developed classifiers are proposed to improve the rate of successful commands to the robotic arm. In one of the implementations, a rate of successful commands up to $91 \%$ was obtained, with wrong commands as low as $1.25 \%$, after 400 attempts to control the robotic arm.

\section{Key Words}

Brain Machine Interface; Neural Networks; Electroencephalograph; Robotic arm; Operant Conditioning; Wavelet Transform; Digital filters; Rehabilitation. 


\section{Sumário}

1. Introdução 14

2. Fundamentos Teóricos 19

2.1. Interfaces cérebro máquina $\quad 19$

2.2. Sinais Eletroencefalográficos. 20

2.3. Condicionamento Operante. 23

2.4. Potenciais Evocados $\quad 25$

2.5. Implantes Corticais 26

2.6. Artefatos 28

2.7. Redes Neurais Artificiais $\quad 29$

2.8. Cinemática de um Manipulador Robótico 31

3. Desenvolvimento de um Eletroencefalógrafo 32

3.1. Descrição 32

3.2. Circuito de Proteção 33

3.3. Amplificação 34

3.4. Right-Leg Driver (RLD) 38

3.5. Conversão Analógico-Digital 39

3.6. Construção do Eletroencefalógrafo. 40

4. Pré-Processamento e Extração de Características 42

4.1. Eliminação do Ruído Remanescente 42

4.2. Filtragem Espacial 44

4.3. Extração de Características 45

4.4. Detecção de Artefatos 50

5. Reconhecimento de Padrões $\quad 51$

5.1. Comitê de Redes Neurais MLP com DPR 52

5.2. Múltiplas Redes Modulares (MRM). 53

5.3. Modelo Hierárquico $(\mathrm{MH})$.

5.4. Implementação de Disparo com MRM. 56

5.5. Implementação Estatística com o Modelo Hierárquico 58 
6. Aplicação da BMI a um Manipulador Robótico 60

6.1. Controle do Manipulador MA-2000 60

6.2. Protocolo de Treinamento. 64

6.3. Uso da Interface. 66

6.4. Experimentos e Resultados 67

7. Conclusões e Trabalhos Futuros 80

$\begin{array}{lr}\text { Bibliografia } & 83\end{array}$

$\begin{array}{lr}\text { Anexos } & 88\end{array}$

Anexol $\quad 89$

Anexo II 98

$\begin{array}{lr}\text { Anexo III } & 100\end{array}$

Anexo IV 102

Anexo V 104

Anexo VI 112 


\section{Lista de figuras}

Figura 1 - Córtex Cerebral [17]. 19

Figura 2 - Aplicações de uma interface cérebro máquina [23-24]. 20

Figura 3 - Sinais eletroencefalográficos durante diferentes atividades [26]. 21

Figura 4 - 10-20 System of Electrode Placement [29]. 21

Figura 5 - Sinal típico associado à Banda Delta em função do tempo (em s). 22

Figura 6 - Sinal típico associado à Banda Theta em função do tempo (em s). 22

Figura 7 - Sinal típico associado à Banda Alpha em função do tempo (em s). 22

Figura 8 - Sinal típico associado à Banda Beta em função do tempo (em s). 23

Figura 9 - Interface cérebro máquina por potenciais evocados 25

Figura 10 - Potencial evocado. 26

Figura 11 - Macaco utilizando uma prótese cortical para se auto-alimentar. $\quad 27$

Figura 12 - Artefatos musculares e oculares em sinais EEG [4]. 28

Figura 13 - Estrutura de uma rede neural $\quad 29$

Figura 14 - Modelo do neurônio artificial. 30

Figura 15 - Cinemática de um manipulador robótico. 31

Figura 16 - Electro-Cap e acessórios. 32

Figura 17 - Diagrama de um canal do eletroencefalógrafo. 33

Figura 18 - Circuito de proteção.

Figura 19 - Amplificador instrumental INA114 - Texas Instruments 35

Figura 20 - Segundo estágio de amplificação. 35

Figura 21 - Terceiro estágio de amplificação. 36

Figura 22 - Diagrama de Bode do amplificador. 37

Figura 23 - RLD para o circuito integrado INA114 38

Figura 24 - RLD para vários canais de amplificação. 39

Figura 25 - NI USB-6009. 39

Figura 26 - Renderização da eletrônica do eletroencefalógrafo. 40

Figura 27 - Eletroencefalógrafo com a caixa metálica aberta. 41

Figura 28 - Sinal EEG na posição Cz. 41

Figura 29 - Resposta em frequência do filtro Butterworth. 43

Figura 30 - Resposta em frequência do filtro Notch. 44

Figura 31 - Sinais estimados pelo método de Hjorth (à direita). 45

Figura 32 - Esquemático da transformada wavelet discreta (DWT). 47

Figura 33 - Árvore de decomposição wavelet discreta para 1kHz. 47

Figura 34 - Transformada wavelet discreta de um sinal EEG. 48

Figura 35 - Comitê de redes MLP com DPR. 52 
Figura 36 - Classificador baseado em múltiplas redes modulares.

Figura 37 - Modelo Hierárquico. 55

Figura 38 - Exemplo de implementação de disparo com MRM. 57

Figura 39 - Exemplo de implementação estatística. 59

Figura 40 - Sistema completo. $\quad 60$

Figura 41 - Manipulador robótico MA2000. 60

Figura 42 - Modelo esquemático do MA2000. 61

Figura 43 - Eletrônica de controle do manipulador robótico. 62

Figura 44 - Parâmetros de Denavit Hartenberg para os três primeiros elos do MA2000. 62

Figura 45 - Cálculo da cinemática inversa para um manipulador de dois elos. 63

Figura 46 - Esquemático 3D dos três primeiros elos do MA2000. 63

Figura 47 - Correspondência das atividades mentais na ativação de um manipulador robótico. 66

Figura 48 - Confiabilidade x Número de sorteios. 69

Figura 49 - Validação da solução analítica para dois padrões, usando o método de Monte Carlo. $\quad 70$

Figura 50 - Confiabilidade x Número de tentativas máximo $\mathrm{N}_{\max }\left(\mathrm{N}_{\min }=5\right) . \quad 71$

Figura 51 - Análise da variação da condição de ocorrência para $N_{\max }=15 . \quad 72$

Figura 52 - Confiabilidade de classificação x Nmin para $\mathrm{N}_{\max }=15$.

Figura 53 - Número de tentativas médio para obtenção de resultado x Nmin. 73

Figura 54 - Histograma das classificações obtidas pelo número de tentativas. 74

Figura 55 - Confiabilidade x Nmin para três padrões. 75

Figura 56 - Experimento com a interface para controle do manipulador. $\quad 76$

Figura 57 - Experimento com o robô móvel: 1- Eletrodos; 2-Computador; 3-

EEG; 4-PCTx Link; 5-Transmissor RF; 6-Robô móvel. 77

Figura 58 - Correspondência das atividades mentais: robô móvel. 78 


\section{Lista de Tabelas}

Tabela 1 - Comparação da influência de parâmetros escolhidos. 49

Tabela 2 - Matriz de confusão do modelo MRM. 54

Tabela 3 - Matriz de confusão do modelo hierárquico. 55

Tabela 4 - Taxas de acerto dos diferentes classificadores. 56

Tabela 5 - Confiabilidade de classificação.

Tabela 6 - Matriz de confusão para três padrões. 75

Tabela 7 - Resultados da aplicação da BMI ao manipulador robótico. 76

Tabela 8 - Resultados da aplicação a um robô móvel. 78 


\title{
Lista de Símbolos e Abreviações
}

\author{
A/D - Analógico / Digital \\ $\mathrm{BMI}$ - Brain Machine Interface \\ CMRR - Common mode rejection rate \\ DC - Direct Current \\ DOF - Degree of Freedom \\ DPR - Driven Pattern Replication \\ DSP - Digital Signal Processor \\ DWT - Discrete Wavelet Transform \\ $E A L$ - Esclerose Amiotrófica Lateral \\ EEG - Eletroencefalograma \\ EMG - Eletromiografia \\ ICSP - In Circuit Serial Programming \\ MLP - Multi Layer Perceptron \\ RAM - Random Access Memory \\ RLD - Right Leg Driver \\ RSM - Ritmo Sensório Motor \\ SCD - Scalp Density Current \\ SCP - Slow Cortical Potential \\ SL - Surface Laplacian \\ SMD - Surface Mounted Device \\ SNA - Sistema Nervoso Autônomo \\ SNC - Sistema Nervoso Central \\ TTL - Transistor Transistor Logic
}

